\title{
DISEÑO Y DESARROLLO DE UN ROBOT AÉREO PARA LA INSPECCIÓN DE COLECTORES DE SANEAMIENTO
}

\author{
José Luis Andrade Pineda \\ Grupo de Robótica, Visión y Control, Universidad de Sevilla, jandrade@us.es \\ Honorio Romero Rodríguez \\ Grupo de Robótica, Visión y Control, Universidad de Sevilla, hromero r@ $@$ hotmail.com \\ Ángel Rodríguez Castaño \\ Grupo de Robótica, Visión y Control, Universidad de Sevilla, castano@us.es \\ Francisco Javier Pérez Grau \\ FADA-CATEC, fjperez@,catec.aero \\ Graciano Carpes Hortal \\ EMASESA, GCarpes@emasesa.com \\ Aníbal Ollero Baturone \\ Grupo de Robótica, Visión y Control, Universidad de Sevilla, aollero@us.es
}

\begin{abstract}
Resumen
Este artículo presenta el diseño y desarrollo de un robot aéreo para la inspección de colectores de saneamiento visitables (con una dimensión mínima de 1.8 metros). Para realizar la inspección de esta infraestructura, en la actualidad se emplean a 5 personas y dos vehículos, y la operación se realiza recorriendo a pie el colector, grabando imágenes durante el recorrido. Esta es una tarea lenta, tediosa y con diversos riesgos para el personal que realiza la inspección. El sistema desarrollado consiste en un UAV que es introducido en el colector y que se alinea y centra en la tubería de forma automática. Desde fuera, un operario puede comandar el UAV avanzando, retrocediendo o parando conforme a las necesidades de la inspección. Dicho operario recibe imágenes en tiempo real conforme el robot se desplaza por la tubería.
\end{abstract}

Palabras clave: robots aéreos, navegación en interiores, inspección de tuberías.

\section{INTRODUCCIÓN}

Existe en la actualidad un incremento muy notable del número de aplicaciones con vehículos aéreos no tripulados (UAVs en su acrónimo anglosajón). Estas aplicaciones implican la realización de tareas en diferentes entornos [14] y para aplicaciones, por ejemplo, relacionadas con el transporte [7], el reparto de mercancías [4] o incluso la cinematografía [8]. En el caso de tuberías o colectores las inspecciones deben realizarse periódicamente para monitorizar, entre otras cosas, los denominados fatbergs. Éstos consisten en acumulaciones de grasa que pueden llegar a obstruir los colectores, como ha ocurrido recientemente en Valencia, Londres o Sidmouth [2].

En el caso de la red de alcantarillado de una ciudad media como Sevilla, su dimensión se acerca a los $3000 \mathrm{~km}$. Siendo una ciudad relativamente plana, la pendiente en estas conducciones es típicamente pequeña y complica el flujo de aguas residuales hacia las estaciones de depuración. Por ello, es muy importante la periódica realización de inspecciones sobre la red, en particular, las de los $220 \mathrm{~km}$ de colectores visitables $(>1.8 \mathrm{~m}$ de diámetro). Los defectos/problemas a detectar incluyen: presencia de obstáculos (véase Figura 1) u obstrucciones, roturas o grietas en la conducción, intrusión de raíces, acometidas ilegales o vertidos no controlados. Para una inspección visual de los mismos, varios operarios deben entrar a estos espacios confinados exponiéndose a riesgos por caída, presencia de gases nocivos, por falta de oxígeno o incluso, riesgos biológicos.

La entrada de robots terrestres a este tipo de espacios para mitigar los riesgos de las personas no es una novedad, sin embargo, para espacios de las dimensiones de los colectores visitables, un robot aéreo puede llegar a ofrecer una mayor flexibilidad. Se trata de un entorno confinado en el que no hay posibilidad de posicionarse usando sistemas globales de navegación por satélite (GNSS en su acrónimo 
anglosajón), y en el que las comunicaciones con la aeronave pueden ser poco fiables. Además, el robot aéreo debería tener un peso y tamaño que permita su fácil transporte hasta el lugar de operación y poder entrar a los colectores a través de los pozos de inspección.

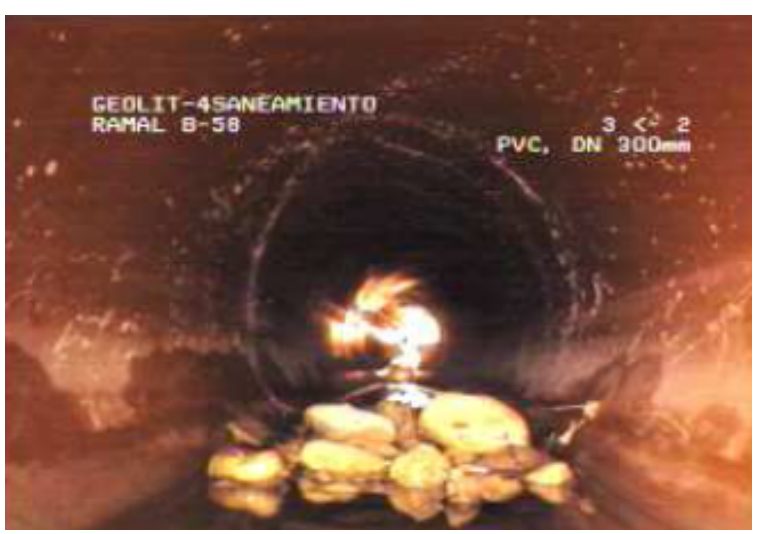

Figura 1: Presencia de obstáculos en colector

En este tipo de entorno cabe destacar el proyecto ARSI [1], parte del $h u b$ europeo ECHORD++, en el que se han realizado diversos experimentos en las alcantarillas de Barcelona. En entornos sin GNSS, aunque no tan reducidos, [9] presenta un pequeño UAV para realizar inspecciones en un entorno industrial cerrado y en [3] se navega en el interior de un buque. También en [12] y [13] se presentan algunos resultados en entornos confinados interiores.

En este artículo se presenta el trabajo realizado para el diseño y desarrollo de un UAV para la inspección del tipo de espacio confinado descrito.

\section{DESCRIPCIÓN DEL PROBLEMA Y REQUISITOS}

\subsection{DESCRIPCIÓN DE LA INSPECCIÓN}

Como se ha indicado anteriormente, el objetivo del sistema es la inspección de los colectores visitables de una red de saneamiento. En este caso, se trata de colectores de forma circular u ovalada que presentan un diámetro mínimo de 1.8 metros y pueden llevar agua hasta una altura de 50 centímetros. Cada tramo de colector tiene una longitud de unos 50 metros y en cada extremo, uniendo los tramos, hay pozos de registro por los que se puede acceder (ver Figura 2).

El robot aéreo debe introducirse por uno de los pozos de acceso, realizar la inspección de un tramo y salir (o ser extraído) por el pozo siguiente. Durante ese recorrido el robot debe grabar imágenes y también debe transmitirlas en tiempo real al operador. En caso de que se detecte la existencia de un defecto o problema, serán los operarios los encargados de solucionarlo accediendo al interior del mismo. De esta forma, el robot permitirá reducir considerablemente la frecuencia de acceso de los operarios a los colectores.

Actualmente los operarios se encargan por completo de la inspección, teniendo que desplazarse en grupos de al menos 2 personas por el interior del colector, con todas las medidas de protección y tiempo que ello conlleva. Con este sistema los operarios sólo tendrán que acceder al interior cuando se haya detectado alguna deficiencia.

\subsection{REQUISITOS DEL ROBOT}

\subsubsection{Dimensiones y Autonomía}

El UAV debe tener el menor peso y tamaño posible, de forma que pueda transportarse fácilmente hasta el lugar de operación y pueda entrar a los colectores a través de los pozos de inspección. La boca de entrada de los pozos presenta una forma circular con un diámetro de 80 centímetros. El tiempo de operación estimado es de 10 minutos por cada segmento a inspeccionar, que típicamente no supera los $50 \mathrm{~m}$.

\subsubsection{Requisitos Operacionales}

Se pretende una inspección semiautomática de la tubería, que no requiera de una pericia especial por parte del operador del UAV. El robot debe desarrollar la operación de la forma más autónoma posible, usando su propio sistema de navegación y evitación de colisiones.

Dadas las características del entorno por el que se debe desplazar (con potenciales obstáculos, ligeras curvaturas en las tuberías, presencia de una lámina de agua y sedimentos que reducen el espacio de vuelo, etc.) se necesita que el UAV sea capaz de posicionarse automáticamente a una altura mínima sobre el suelo o la lámina de agua, y que mantenga al mismo tiempo una distancia de seguridad o separación mínima con las paredes y el techo. Como referencia, se fija una tubería de forma circular y diámetro 1.80 metros por el que discurre una lámina de agua de hasta 0.5 metros.

En cuanto al desplazamiento a lo largo de la tubería, el robot debe detenerse, avanzar o retroceder según le indique el operador, y en función de las imágenes recibidas en el puesto de mando. Se ha determinado que no es necesario conocer el desplazamiento longitudinal en el colector de forma precisa. Además, el robot debe tener un comportamiento seguro y fiable ante una posible pérdida de comunicaciones. 


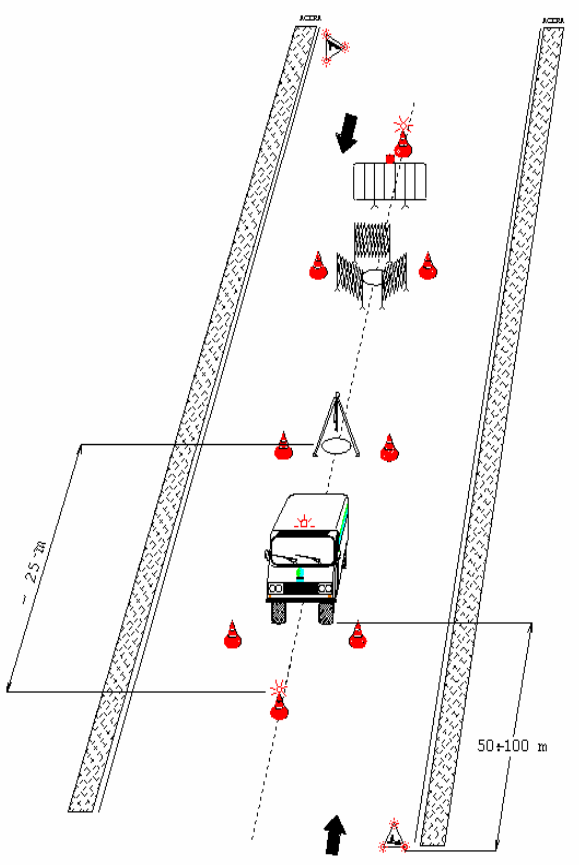

Figura 2: Procedimiento tipo en el acceso por pozo de inspección al Colector: Corte, Señalización y uso de vehículo para traslado y como Puesto Base

Por último, la identificación de defectos y fallos deben realizarla los operarios a partir de imágenes con calidad y resolución suficientes. Dadas las condiciones de iluminación en el interior del colector, se deberá disponer de un sistema de iluminación artificial que permita obtener imágenes de calidad (al menos PAL), así como un sistema de grabación y transmisión de vídeo.

\subsection{SOLUCION PROPUESTA}

Como se ha expuesto anteriormente, el objetivo es permitir que el operario se centre en la interpretación de las imágenes que el UAV captura con las cámaras de a bordo, y que no requiera de una pericia y entrenamiento especial en el manejo del robot.

Para ello, se ha dotado al operador de un gamepad con el que controlar el robot. Uno de los sticks del gamepad envía consignas de desplazamiento longitudinal al robot, mientras que el sistema de navegación del UAV lo mantiene alineado, centrado y a una altura prefijada sobre el suelo. Para esto se decide emplear sensores de distancia. En concreto, se propone el uso de sensores de distancia, dispuestos de forma que se puede determinar la orientación del UAV respecto a la tubería y la distancia a las paredes de la misma.

Respecto a la previsible falta de fiabilidad de las comunicaciones en los colectores, se decide usar un mecanismo de watch-dog, de forma que ante una pérdida de comunicaciones puntual con el operador (un puesto base en la calle), el UAV mantendrá la posición hasta que las comunicaciones se recuperen. En caso de pérdida de comunicaciones prolongada, el UAV deberá volver a su punto inicial (o hasta recuperar las comunicaciones) de forma segura.

En cuanto a la baja o nula luminosidad de este entorno, se emplearán luces tipo LED que permitan una visibilidad suficiente. Las imágenes serán enviadas en tiempo real al operario mediante un sistema de transmisión de video analógico $\mathrm{y}$, al mismo tiempo, serán grabadas a bordo (sin pérdida de calidad debido a la transmisión). Se ha seleccionado un transmisor de video analógico en lugar de uno digital debido a que ante interferencias el transmisor analógico permite tener imágenes con calidad degradada, mientras que el digital no permitiría disponer de ninguna imagen. Además, los posibles retrasos en la transmisión del vídeo suponen un factor clave para el éxito de la inspección remota, por lo que se ha optado por un sistema analógico de muy baja latencia.

Por último, en cuanto a una posible presencia de atmósferas ATEX, el robot incorpora un sensor de gases que permite apagarlo por completo en caso de detectarse ese tipo de atmósfera.

\section{DESARROLLO DEL ROBOT AÉREO}

\subsection{HARDWARE PARA NAVEGACIÓN}

Dado que las bocas de acceso tienen una dimensión de $800 \mathrm{~mm}$ se ha usado como base del robot aéreo el chasis F550 de la empresa DJI. A esta base mecánica se le ha incorporado un autopiloto Pixhawk (ver Figura 3). Esta controladora se encarga de la estabilización angular y la altura del UAV.

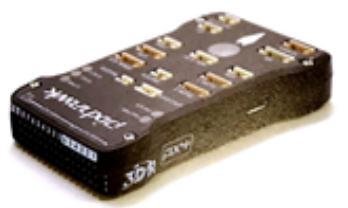

PIXHAWK

Procesador: STM32F427 (32-bit, 225MIPS)

Sensores: 3 giroscopios, 3 acelerómetros, barómetro

Compatible con: magnetómetro, GPS, sónar, telemétrica. El Pixhawk es un sistema potente capaz de transmitir telemetría o de grabarla en su tarjeta de memoria interna potente capaz de transmitir telemetría o de grabarla en su tarjeta de memoria interna
MicroSD. Existen diversos sistemas de control, incluido el plloto automático completo.

Figura 3: Controladora de vuelo del UAV para inspección de colectores

Una vez dotado el frame F550 de un autopiloto completo en el que se sustenten las funciones de 
control de bajo nivel en el vuelo del UAV, es necesario seleccionar el hardware sobre el que ejecutar el algoritmo de navegación que permite mantener centrado y alineado el robot, así como el resto de funcionalidades de la inspección. Tras varias pruebas de rendimiento, se ha seleccionado una tarjeta Raspberry $\mathrm{Pi}$ para implementar estas funcionalidades.

En esa tarjeta Raspberry Pi se implementan las funciones utilizando el framework ROS (Robot Operating System) [10]. De esta forma se puede hacer uso de todas las librerías y paquetes ya existentes para aplicaciones robóticas, que en particular simplificarán las tareas de adquisición de datos de los distintos sensores.

Por último, es necesario definir los sensores que permitirán centrar el UAV en la tubería del colector, como la mejor estrategia para garantizar la seguridad. Esta zona es la más diáfana dentro de la tubería: por abajo puede haber una lámina de agua y también sedimentos, y por arriba podría haber diversos colgajos.

\subsubsection{Sensores para Posicionamiento}

Se ha seleccionado la tecnología de infrarrojos de tiempo de vuelo (TOF), por ser mucho más rápida que los sensores de ultrasonido y mucho más pequeña y ligera que otros sistemas basados en láser. En el mercado existen gran variedad de estos sensores con buen soporte ROS.

En este caso, existía la particularidad de que los sensores deberían dar una medida aceptable incluso apuntando sobre agua. Por ello, se han realizado una serie de pruebas con varios de ellos (Lightware SF11 y TeraRanger One). Tras ellas, el sensor elegido es el TeraRanger One Type A, por su pequeño tamaño, alto rendimiento y bajo consumo de energía.

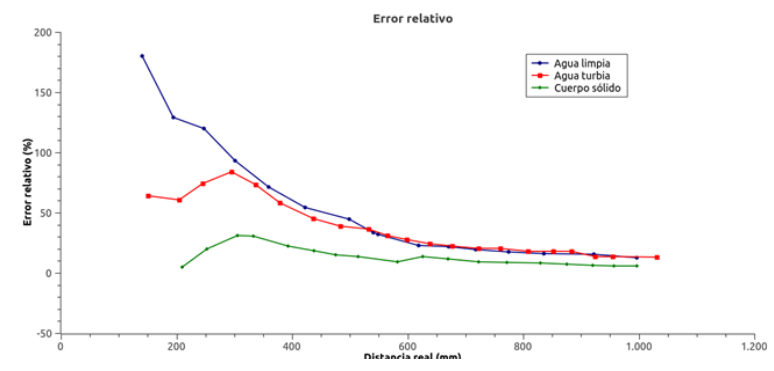

Figura 4: Medición de altura sobre lámina de agua limpia de $12.5 \mathrm{~cm}$ con sensor TeraRanger One

Además, en pruebas específicas sobre agua con distintos niveles de turbidez es el que mejor rindió. Este sensor tiene rango de utilidad entre los $200 \mathrm{~mm}$ y los $14 \mathrm{~m}$, y de las pruebas realizadas, se aprecia que por encima de los $600 \mathrm{~mm}$ (véase Figura 4) la medición de altura es estable sobre agua y apenas depende del nivel de turbidez, aunque en comparación a lo que daría sobre un cuerpo sólido (en la gráfica se usó un trozo de madera) es necesario tomar en cuenta la existencia de un pequeño offset.

De forma adicional, se disponen dos de estos sensores en el plano del UAV, para poder detectar las paredes laterales. Aunque la configuración que inicialmente se pensó disponía ambos de forma lateral a $0^{\circ}$ y $180^{\circ}$ respecto del eje OY local, después se ha visto que era más interesante anticiparse a posibles obstáculos que fueran apareciendo en el recorrido y por ello se modificó a $20^{\circ}$ y $160^{\circ}$ respecto del mismo eje OY. Esta variación de $20^{\circ}$ aseguraba que, si algo se oponía al movimiento frontal del UAV, éste pudiera reaccionar antes de una posible colisión.

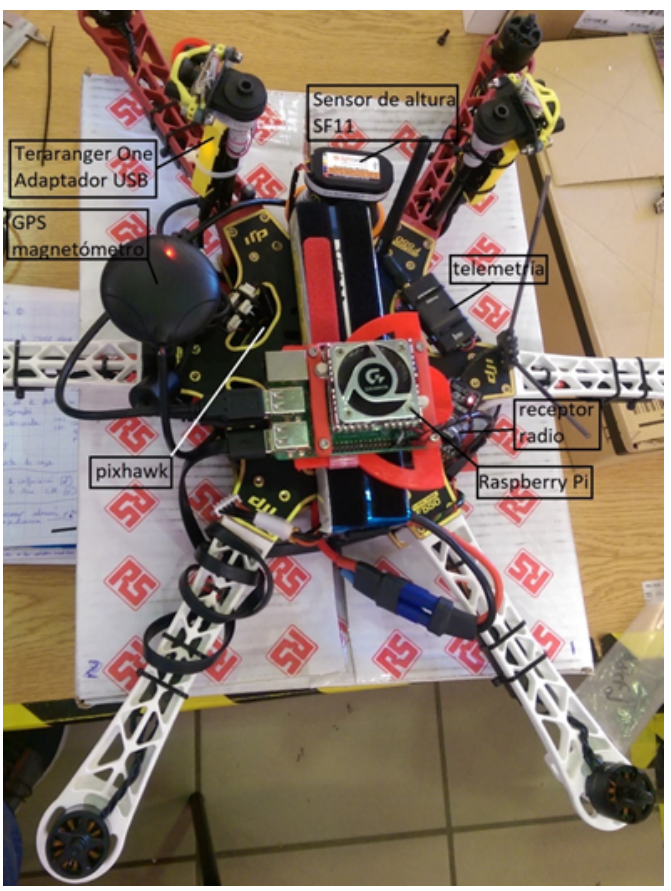

Figura 5: Elementos hardware para una navegación en el interior de colectores de saneamiento

\subsection{ELEMENTOS DE FLOTACIÓN Y PROTECCIÓN}

El nivel de agua en las tuberías a inspeccionar puede ser considerable, según el estado de limpieza de los colectores o ante posibles lluvias y otros eventos que aumentan el caudal de agua en el sistema de canalizaciones. Por esta razón es importante que el UAV tenga cierta protección ante la posibilidad de caídas o amerizajes sobre el agua, además de evitar el efecto de las posibles salpicaduras que puedan ocurrir por el flujo de aire. Por otro lado, como cualquier 
obstáculo que haya bajo las hélices reduce el rendimiento y la sustentación, se ha de diseñar el sistema de flotabilidad (véase Figura 10) que no dificulte la impulsión de los motores. De igual manera, en la parte inferior se ha diseñado e impreso una carcasa plástica para la protección de los circuitos. Aunque no se evita del todo la posibilidad del paso de agua, sí que la dificulta mucho. También se han protegido las hélices de posibles contactos con objetos con protecciones plásticas en el plano horizontal.

\subsection{CAPTACIÓN Y TRANSMISION VÍDEO}

Aunque en pruebas iniciales se barajó el uso de focos tipo linterna, finalmente para el sistema de iluminación a usar en colectores se optó por utilizar tiras LED que minimizan en gran medida el consumo de potencia mientras se consigue unas características de iluminación del entorno suficiente. Para el sistema de vídeo que permita la inspección visual del colector, se propone usar una solución basada en pequeñas cámaras empleadas típicamente en aplicaciones de vuelo en primera persona (FPV). Con el objetivo de visionar las paredes laterales del colector a medida que el UAV avanza en la misión, se han integrado dos pequeñas cámaras RunCam Split 2S. La orientación de cada una es la adecuada para cubrir los laterales del colector, y además sus campos de visión se superponen en la vista central en el eje de dicho colector. De esta manera, se puede controlar el avance longitudinal de la inspección. Cada cámara está conectada a un pequeño transmisor de vídeo y de potencia regulable, de manera que nos permita testear distintos niveles de potencia para alcanzar la recepción de vídeo deseada. De la misma forma, las cámaras están conectadas a un sistema de grabación de vídeo a bordo del UAV (ver Figura 6), conocido como DVR (Digital Video Recorder). Esto permitirá el visionado de la inspección a posteriori, especialmente en los casos en los que por alguna razón se pierda puntualmente la comunicación inalámbrica con la estación de control.

En el puesto de control al nivel de calle se contará con un receptor de vídeo por cada cámara, conectado a un monitor que permita el visionado en tiempo real de todo lo que está ocurriendo en el entorno del UAV.

\section{DESARROLLO DEL SOFTWARE DE NAVEGACIÓN}

Además de los paquetes estándar proporcionados por ROS, se ha empleado la librería abierta UAL (UAV Abstraction Layer). UAL [11] es una librería abierta desarrollada por el GRVC para simplificar los interfaces con los UAVs a través de una capa de abstracción que unifica la forma de interaccionar con autopilotos de diversos fabricantes. Así, su objetivo es simplificar el desarrollo y las pruebas de algoritmos de alto nivel en robótica aérea. UAL puede funcionar con UAVs simulados o reales y proporciona llamadas para emitir comandos tales como despegue, aterrizaje y consignas de control en posición y velocidad.

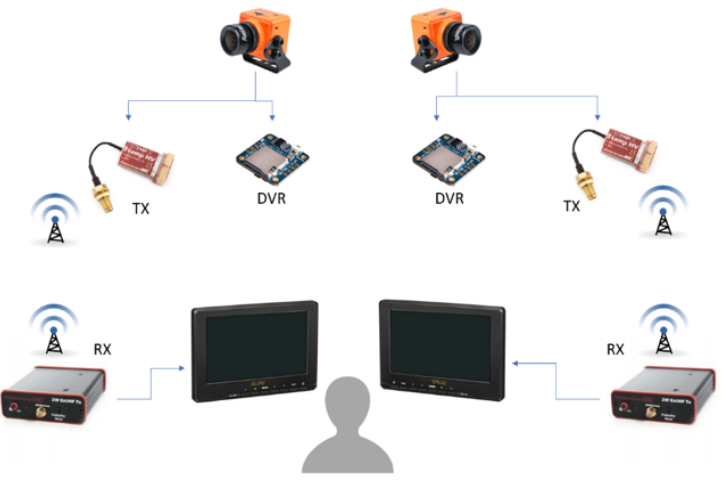

Figura 6: Diagrama del Sistema de Transmisión de Vídeo

A continuación, se detallan las tareas que se han acometido para implementar las funciones de navegación requeridas en el sistema mostrado en la Figura 5. Dado que la plataforma F550 es bastante común, existen parametrizaciones previas con las que inicialmente se obtendría buen comportamiento. Lo más importante es un correcto ajuste de los PIDs de estabilización de ángulos y control en velocidad. En este caso también ha sido necesario retocar los parámetros de geometría y pesos del UAV. Para este ajuste se ha usado la aplicación QGroundControl, por su facilidad para instalar el software de navegación, para explotación básica del Pixhawk [6] sobre el hexacóptero. Desde la QGroundControl también se ha ajustado el control manual desde la emisora, los sensores, modos de vuelo, configuración de la batería, etc.

\subsection{SIMULACIÓN GAZEBO}

Se ha empleado el simulador Gazebo [5] para realizar una primera validación del sistema: el robot aéreo F550 es emulado y publica la información de sus sensores en mensajes de ROS. Además, el propio F550 emulado recibe órdenes de la lógica y el control que estamos simulando.

En la Figura 7 se muestra cómo se ha evaluado el comportamiento de la plataforma mostrada en la Figura 5, haciendo uso de Gazebo. En nuestro caso, se diseñó con SolidWorks un modelo lo más cercano y realista posible al F550 para obtener respuestas similares a la realidad, incluyendo un modelo $3 \mathrm{D}$ de la tubería. 


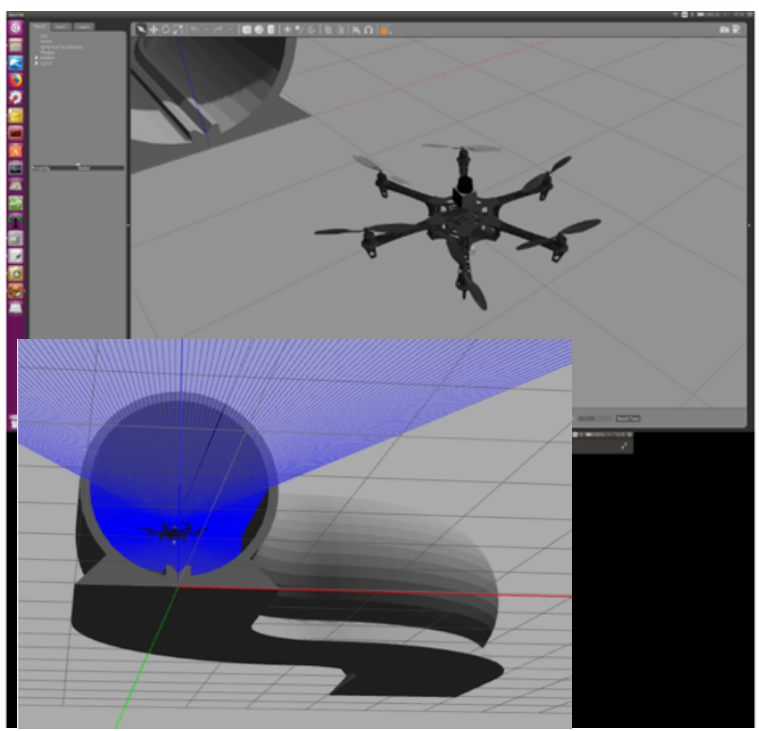

Figura 7: Simulación Gazebo de navegación en base a dos sensores láser en el plano de avance del F550

$(\mathrm{XY})$ y uno en altura $(\mathrm{Z})$

\subsection{MECANISMO DE ORIENTACIÓN Y ALINEAMIENTO EN TUBERÍA}

La navegación dentro de la tubería sigue el esquema funcional que se muestra en la Figura 8, donde se representa el UAV con sensor de altura (cuadrado) y los dos sensores de avance (pentágonos). En nuestro entorno de desarrollo la comunicación se realiza mediante la publicación de mensajes en tópicos ROS, lo que nos permite simular muchos de los dispositivos con los que nuestro sistema trabaja, de forma que podemos aislar la funcionalidad de nuestro sistema del código de comunicación entre las diferentes partes del sistema, sensores y actuadores.

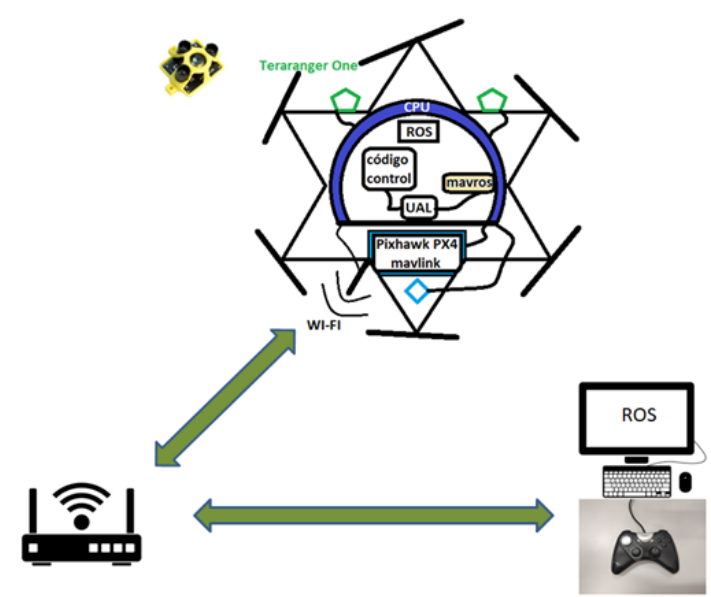

Figura 8: Esquema funcional del entorno de desarrollo con que testar el Sistema de Navegación
Uno de los aspectos clave al desarrollar una aplicación como este sistema de navegación es la capacidad de repetir los experimentos y poder simular los sensores y actuadores antes de realizar las pruebas de campo. Una vez validado el funcionamiento en el simulador, se han desarrollado pruebas de campo tanto en los laboratorios del GRVC, en el testbed de interiores de FADA-CATEC y en el Centro de Formación del Agua (CEFORA) de EMASESA, sobre una conducción cilíndrica real.

\subsubsection{Desarrollo del Sistema de Control}

El objetivo fundamental de este control es proporcionar una navegación asistida y segura dentro de colectores cilíndricos de sección aproximadamente constante, dando la opción de avanzar o retroceder a lo largo del mismo.

Los tres sensores proporcionan medidas a la CPU de a bordo de la estimación de dos puntos en el plano horizontal y uno en el plano vertical. Con los dos primeros (véase Figura 9), el UAV es capaz de orientarse y alinearse permitiendo el avance a lo largo del eje del colector y asistiendo cuando sea necesario para evitar colisiones, además de poder grabar video.

Sin embargo, hay que tener en cuenta que, al iniciar el posicionamiento en el comienzo de misión de un modo automático, el UAV desconoce por completo la orientación; el código en ejecución en la Raspberry Pi carece de esa información previa. Por ello, se ha configurado que, en esta primera fase del alineamiento de la aeronave, el F550 gire varias veces un cierto ángulo alternativamente a izquierda y derecha, con el objetivo de buscar la distancia mínima en la suma de los datos captados por lo sensores. Cuando detecta y verifica esta distancia mínima, el multirrotor ya está orientado según la generatriz del cilindro. Al mismo tiempo que se busca la orientación en la generatriz, se comandan movimientos laterales en $\mathrm{OY}$ de ejes locales del UAV, de tal manera que la lectura del sensor izquierdo, sea igual que la lectura del sensor derecho, lo que se traduce en una aproximación al eje central del plano que corta al colector a una altura prefijada.

Al terminar la fase de centrado y de alineado, el controlador intenta mantener en esa posición al UAV y si por alguna razón (por ejemplo, por efecto del flujo de aire) sale de esta posición más de lo debido, se itera de nuevo el proceso hasta volver a conseguir el objetivo. Por debajo de estos límites existe un control en rampa, tanto para el centrado como para el alineado. 


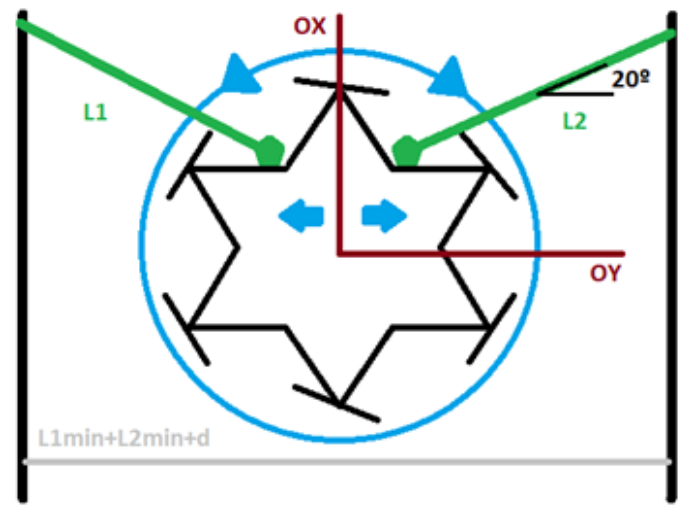

Figura 9: Alineación al inicio de la misión en base a los sensores laterales

\section{EXPERIMENTACIÓN}

En la Figura 10 se muestra una imagen de los primeros tests de campo en que se ha puesto a prueba la solución de navegación desarrollada.

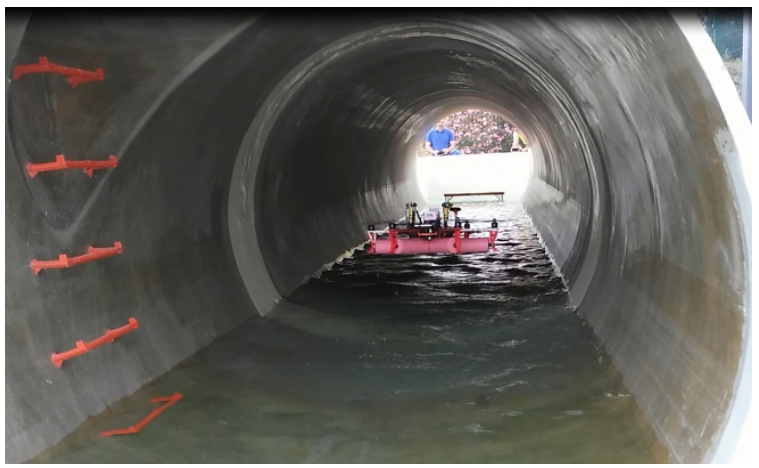

Figura 10: Pruebas sobre lámina de $40 \mathrm{~cm}$ de agua, con ajuste en altura de $30 \mathrm{~cm}$ sobre la misma

La Figura 11 muestra los primeros resultados con el sistema de captación y transmisión de vídeo.

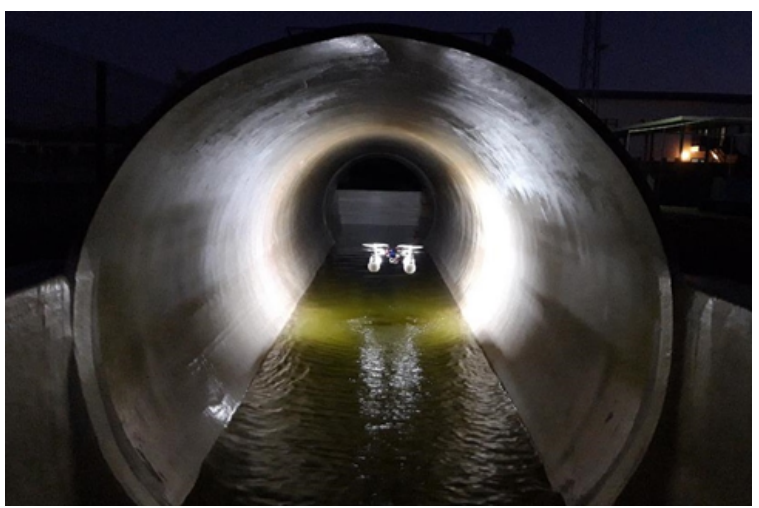

Figura 11: Pruebas del Sistema de Iluminación

\section{CONCLUSIONES}

De la experimentación se pueden extraer varias conclusiones:

- Según los experimentos en campo, el sistema de iluminación y de transmisión de vídeo cumple con los requerimientos del caso de uso.

- Según el estudio respecto a las necesidades de navegación autónoma y al conjunto de test realizados en campo, se comprobó que utilizar un sensor de rango como sensor de altura sí cumple con las necesidades planteadas.

- En cuanto al uso de los dos sensores para ubicar al UAV dentro del colector de manera alineada y centrada, se puede concluir que satisface de forma aceptable su cometido. Sin embargo, aparecen oscilaciones al vibrar en torno al punto de equilibrio.

- Dado que la tasa de muestreo de los sensores de rango puede alcanzar los $1000 \mathrm{~Hz}$, se tuvo que realizar overclocking en la Raspberry $\mathrm{Pi} y$ fue necesario añadir un ventilador para su refrigeración. A los problemas de calentamiento también se añadía la pérdida de eficacia en el control de la plataforma debido a retrasos. La utilización de una tarjeta microcontroladora más potente deberá incorporarse a futuro.

Para un trabajo futuro, se utilizará una tarjeta microcontroladora más potente y se usará un tercer sensor en el plano horizontal, de modo que consigamos desacoplar los procesos de alineado y de centrado y consigamos en ambos procesos un comportamiento más estable.

\section{Agradecimientos}

Este trabajo ha sido parcialmente financiado por el Centro para el Desarrollo Tecnológico Industrial (CDTI) y la Corporación Tecnológica de Andalucía (CTA). También agradecemos a Rafael Salmoral su colaboración en las pruebas de vuelo. 


\section{English summary}

\section{DESIGN AND DEVELOPMENT OF AN AERIAL ROBOT FOR SEWER INSPECTION}

\begin{abstract}
This paper presents the design and development of an aerial robot for sewer inspections. This kind of inspection requires at least two workers to get inside the sewer and walk along while recording a video. This work is slow, tedious and risky. The proposed aerial robot can be placed inside the pipe and it autonomously centers and aligns itself inside the pipe. Then, an outside worker can command the aerial robot to go forward, backward or stop when needed. The operator receives real-time images while commanding the robot.
\end{abstract}

Keywords: aerial robotics, indoor navigation, sewer inspection.

\section{Referencias}

[1] ARSI project: https://eurecat.org/en/portfolioitems/aerial-robot-for-sewer-inspection/

[2] BBC News (Online version), https://www.bbc.com/news/ukenglanddevon46787461

[3] Bonnin-Pascual,F., Garcia-Fidalgo,E., Ortiz,A.: Semi-autonomous visual inspection of vessels assisted by an unmanned micro aerial vehicle. 2012 IEEE/RSJ International Conference on Intelligent Robots and Systems. pp. 3955-3961 (2012).

[4] Dorling, K.; Heinrichs, J.; Messier, G.G.; Magierowski, S. "Vehicle Routing Problems for Drone Delivery", IEEE Trans. Syst. Man Cybern. Syst. 47, 70-85 (2017).

[5] Furrer F., M. Burri, M. Achtelik, and R. Siegwart, "Robot operating system (ROS): the complete reference (volume 1)", Springer International Publishing, ch. RotorS-A modular Gazebo MAV simulator framework, pp. 595-625 (2016).

[6] Meier L., T. Gubler, J. Oes, D. Sidrane, D. Agar, B. King, A. Babushkin, px4dev, M. Charlebois, R. Bapst, et al., "Px4/firmware: v1.7.3 stable release," (2018).
[7] Mellinger, D.; Shomin, M.; Michael, N.; Kumar, V. "Cooperative Grasping and Transport Using Multiple Quadrotors", Distributed Autonomous Robotic Systems: The 10th International Symposium; Martinoli, A., Mondada, F., Correll, N., Mermoud, G., Egerstedt, M., Hsieh, M.A., Parker, L.E., Støy, K., Eds.; Springer: Berlin/Heidelberg, Germany; pp. 545-558 (2013).

[8] Nägeli, T.; Meier, L.; Domahidi, A.; AlonsoMora, J.; Hilliges, O. "Real-time Planning for Automated Multi-view Drone Cinematography", ACM Trans. Graph., 36, 132:1-132:10 (2017).

[9] Nikolic,J., Burri,M., Rehder,J., Leutenegger,S., Huerzeler,C., Siegwart,R.: A UAV system for inspection of industrial facilities. 2013 IEEE Aerospace Conference. pp.1-8 (2013).

[10] Quigley M., K. Conley, B. Gerkey, J. Faust, T. Foote, J. Leibs, R. Wheeler, and A. Y. $\mathrm{Ng}$, "ROS: an open-source Robot Operating System," ICRA Workshop on Open Source Software, vol. 3, no. 3.2. Kobe, Japan (2009).

[11] Real F., Torres-González A., Ramón-Soria P., Capitán J. and Ollero A., "UAL: An Abstraction Layer for Unmanned Aerial Vehicles", The 2nd International Symposium on Aerial Robotics, pp. 100-106 (2018).

[12] Shen,S., Michael,N., Kumar,V.: Obtaining Liftoff Indoor: Autonomous Navigation in Confined Indoor environments. IEEE Robotics \& Automation Magazine 20 (4), 40-48 (2013).

[13] Tripicchio,P., Satler,M., Unetti,M., Avizzano, C.A.: Confined spaces industrial inspection with microaerial vehicles and laser range finder localization. International Journal of Micro Air Vehicles 10 (2), 207-224 (2018).

[14] Visiongain, "The unmanned aerial vehicles (UAV) market 2011-2021:Technologies for ISR and counter-insurgency," Visiongain, Tech. Rep. (2011).

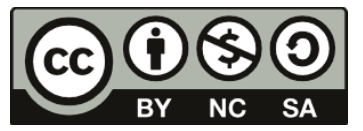
(C) 2019 by the authors. Submitted for possible open access publication under the terms and conditions of the Creative Commons Attribution CC BY-NC-SA 4.0 license (https://creativecommons.org/licenses/bync-sa/4.0/deed.es). 